\title{
Energy quantisation and time parameterisation
}

\author{
Alon E. Faraggi ${ }^{1}$, Marco Matone ${ }^{2, a}$ \\ ${ }^{1}$ Department of Mathematical Sciences, University of Liverpool, Liverpool L69 7ZL, UK \\ 2 Dipartimento di Fisica e Astronomia "G. Galilei" and Istituto Nazionale di Fisica Nucleare, Università di Padova, Via Marzolo 8, \\ Padova 35131, Italy
}

Received: 11 November 2013 / Accepted: 9 December 2013 / Published online: 23 January 2014

(C) The Author(s) 2014. This article is published with open access at Springerlink.com

\begin{abstract}
We show that if space is compact, then trajectories cannot be defined in the framework of the quantum Hamilton-Jacobi (HJ) equation. The starting point is the simple observation that when the energy is quantised it is not possible to make variations with respect to the energy, and the time parameterisation $t-t_{0}=\partial_{E} \mathcal{S}_{0}$, implied by Jacobi's theorem, which leads to the group velocity, is ill defined. It should be stressed that this follows directly from the quantum $\mathrm{HJ}$ equation without any axiomatic assumption concerning the standard formulation of quantum mechanics. This provides a stringent connection between the quantum $\mathrm{HJ}$ equation and the Copenhagen interpretation. Together with tunnelling and the energy quantisation theorem for confining potentials, formulated in the framework of quantum HJ equation, it leads to the main features of the axioms of quantum mechanics from a unique geometrical principle. Similar to the case of the classical HJ equation, this fixes its quantum analog by requiring that there exist point transformations, rather than canonical ones, leading to the trivial hamiltonian. This is equivalent to a basic cocycle condition on the states. Such a cocycle condition can be implemented on compact spaces, so that continuous energy spectra are allowed only as a limiting case. Remarkably, a compact space would also imply that the Dirac and von Neumann formulations of quantum mechanics essentially coincide. We suggest that there is a definition of time parameterisation leading to trajectories in the context of the quantum $\mathrm{HJ}$ equation having the probabilistic interpretation of the Copenhagen School.
\end{abstract}

\section{Introduction}

The validity of quantum mechanics is indisputable, but it leaves many conceptual problems unresolved. For this reason over the years numerous schemes have been proposed to

\footnotetext{
a e-mail: matone@pd.infn.it
}

address such issues. Among those, the quantum HamiltonJacobi (HJ) theory is one of the most investigated topics; see [1-8] for a partial list of papers. These studies involve the foundation of quantum mechanics, in particular its interpretation, cosmology, the analysis of quantum dynamics, molecular trajectories, etc. It has been suggested that the quantum Hamilton-Jacobi equation (QHJE) yields a trajectory representation of quantum mechanics (see e.g. $[9,10]$ and references therein). The absence of trajectories is, however, inherent in the Copenhagen probabilistic interpretation of the quantum mechanical wave function. There appears, therefore, to be a fundamental dichotomy between the two approaches. In this paper we offer a solution to this puzzle. We demonstrate the absence of trajectories in the derivation of the QHJE from point transformations leading to the trivial hamiltonian [11-18]. The basic point is that trajectories can only be defined by time parameterisation of them, and include the Bohm-de Broglie pilot wave representation and Floyd's time parameterisation [19-27] by using Jacobi theorem. We show in this paper that these time parameterisations are ill defined. This resolves the dichotomy with the Copenhangen interpretation.

In [28], we considered a Legendre duality in the framework of the Schrödinger equation. Consistency of this mathematical structure in the context of the phase space reveals that classical mechanics must be modified [11-17]. The main idea has been to implement, as in the derivation of the classical HJ equation, the transformations leading to the free state. The difference is that we did not consider the usual canonical transformation with the space coordinate $q$ and the conjugate momentum $p$ considered as independent variables. Rather, we performed the transformation on $q$ and considered the transformation on $p$ as the one induced by the coordinate transformation, by assuming that the analog of the Hamilton characteristic function transforms as a scalar field. Such a transformation leads to a basic cocycle condition which, in turn, implies that the analog of the Hamilton characteristic function must satisfy the quantum analog of the stationary $\mathrm{HJ}$ 
equation. The derivation extends to the higher dimensional non-stationary case and to the relativistic case as well [18].

A basic theorem in [11-17] is that energy quantisation for bound states follows as a consistency condition. We stress that, whereas in the standard approach energy quantisation is a direct consequence of the wave-function interpretation, here it follows from the cocycle condition without any assumption on the meaning of the wave function.

The quantum Hamilton-Jacobi equation is

$\frac{\partial \mathcal{S}}{\partial t}+\frac{1}{2 m}(\nabla \mathcal{S})^{2}+V-\frac{\hbar^{2}}{2 m} \frac{\Delta R}{R}=0$

$\frac{\partial R^{2}}{\partial t}+\frac{1}{m} \nabla \cdot\left(\nabla R^{2} \mathcal{S}\right)=0$,

which can be obtained by identifying a solution of the Schrödinger equation with $\operatorname{Re}^{\frac{i}{\hbar} \mathcal{S}}$. Note that $\mathcal{S}$ is the quantum analog of Hamilton's principal function. Let us now consider a particle in a stationary state with energy $E$. We have

$\mathcal{S}=\int p \cdot \mathrm{d} q-E\left(t-t_{0}\right)$,

where the first term, which we denote by $\mathcal{S}_{0}$, is the quantum analog of Hamilton's characteristic function. Let us now assume that such a particle admits a trajectory $q=q(t)$. Performing a variation of time and energy, keeping fixed the initial and final spatial coordinates of the trajectory, we have

$\delta \mathcal{S}=\frac{\delta \mathcal{S}_{0}}{\delta E} \delta E-\left(t-t_{0}\right) \delta E-E \delta t$,

and by Eq. (3)

$t-t_{0}=\frac{\partial \mathcal{S}_{0}}{\partial E}$.

This is the time parameterisation of particle trajectories that, as first observed by Floyd [19-27], should be used in considering the quantum HJ equation. This is just how trajectories are defined in CM as it implies the group velocity.

A simple but basic initial observation is that in the case of quantised spectra it is not possible to make the variation of the energy. In particular, the trajectories $q=q(t)$, which would follow by inverting Eq. (4), do not exist in the case of discrete energy spectra. Consistency arguments also show that this excludes the possibility of using Eq. (4) even in the case of continuous energy spectra. It is clear that the non-existence of trajectories suggests that also the probabilistic interpretation may be derived without imposing it as a basic axiom.

As we will see, there is a connection between the nonexistence of trajectories and the cocycle condition. In particular, we will see that the implementation of the cocycle condition fixes gluing conditions on the ratio of two linearly independent solutions of the wave function that can be satisfied only on compact spaces, so that continuous energy spectra arise only in the decompactification limit. The fact that such gluing conditions are a basic step in the geometrical formulation of [11-18], related to Legendre duality [28], and the fact that they led to the quantisation of the energy in the case of confining potentials, suggest that they should be always satisfied. It should be observed that in the case of a free particle the level spacing of the energy spectrum will be determined by the geometry of our three-dimensional space, essentially of the order $R^{-2}$, where $R$ is some characteristic cosmological length, and therefore extremely tiny. This, of course, does not mean that such a spacing could not be detected a priori. The fact that trajectories cannot be well defined provides an intriguing relation between quantum $\mathrm{HJ}$ theory and the Copenhagen interpretation. However, the reason why they do not exist in the quantum HJ theory is that it is just the time parameterisation that does not exist. In some sense a similar view is also the one of the Copenhagen interpretation. Actually, time is not a (self-adjoint) operator, so that also in standard quantum mechanics time is not an observable.

There are additional reasons to consider the possibility that a free particle may have a quantised energy spectrum, even if the level spacing is extremely tiny. A rigourous treatment of continuous spectra requires elaborated structures and the probability of finding the particle in a given volume cannot be defined. Furthermore, it should be observed that there are two formulations of quantum mechanics in the framework of the Copenhagen interpretation. We have the one by von Neumann [29], where quantum states are always rays in the Hilbert space $L^{2}(\mathbb{R})$, and the one by Dirac [30], which in general requires a rigged Hilbert space and eigenfunctionals. In particular, in the Dirac formulation, the wave function of a free particle in $\mathbb{R}^{3}$ is seen as a tempered distribution, that is, an element in $\mathcal{S}^{\prime}\left(\mathbb{R}^{n}\right)$, the dual space of the Schwarz space $\mathcal{S}\left(\mathbb{R}^{n}\right)$. In the case of compact spaces the Helmholtz equation $-\frac{\hbar^{2}}{2 m} \Delta \psi=E \psi$

has only a discrete spectrum, so that the two formulations would essentially coincide. More generally, a compact space would fix some natural cutoff that may play an important rôle. We also consider the problem of defining a sort of probabilistic time such that the resulting trajectories in the quantum HJ theory reproduce the probabilistic interpretation of the Copenhagen School.

\section{The cocycle condition and energy quantisation}

We define

$\mathcal{W}(q)=V(q)-E$,

where $V$ is the potential energy and $E$ the energy level. The main point of [11-17] is to assume the existence transforma- 
tions leading any system to the one with trivial hamiltonian, that is, with $\mathcal{W}(q)=0$. Doing this in CM leads to the classical HJ equation. The difference in the approach of [11-17] is that, whereas in CM one considers the canonical transformations, where $p$ and $q$ are considered independent, we considered point transformations. More precisely, we considered the transformation of $q$ and fixed the transformation on the conjugate momentum as the one induced by the relation $p=\partial_{q} \mathcal{S}_{0}$, by considering $\mathcal{S}_{0}$, the analog of the Hamilton characteristic function, as a scalar function under such a transformation of $q$. The derivation can be extended to the non-stationary case and to the relativistic version as well [18]. Therefore, the derivation of $[11,18]$ is based on the principle that all physical states labelled by the function $\mathcal{W}(q)$ can be connected by a coordinate transformation,

$q^{a} \rightarrow q^{b}=q^{b}\left(q^{a}\right)$

defined by

$\mathcal{S}_{0}^{b}\left(q^{b}\right)=\mathcal{S}_{0}^{a}\left(q^{a}\right)$.

This implies that there always exists a coordinate transformation connecting any physical state to the one with $\mathcal{W}^{0}\left(q^{0}\right)=$ 0 . Inversely, this means that any physical state can be reached from the one with $\mathcal{W}^{0}\left(q^{0}\right)=0$ by a coordinate transformation. This cannot be consistent with CM. The reason is that in CM the physical system with $\mathcal{W}^{0}\left(q^{0}\right)=0$ remains a fixed point under coordinate transformations. Thus, in CM it is not possible to generate all systems by a coordinate transformation from the trivial one. This implies a modification of CM, which is analysed by adding a still unknown function $Q(q)$ to the classical HJ equation. Consistency conditions then fix the transformation properties for $\mathcal{W}(q)$,

$\mathcal{W}^{v}\left(q^{v}\right)=\left(\partial_{q^{v}} q^{a}\right)^{2} \mathcal{W}^{a}\left(q^{a}\right)+\left(q^{a} ; q^{v}\right)$

and

$Q^{v}\left(q^{v}\right)=\left(\partial_{q^{v}} q^{a}\right)^{2} Q^{a}\left(q^{a}\right)-\left(q^{a} ; q^{v}\right)$,

which fixes the cocycle condition

$\left(q^{a} ; q^{c}\right)=\left(\partial_{q^{c}} q^{b}\right)^{2}\left[\left(q^{a} ; q^{b}\right)-\left(q^{c} ; q^{b}\right)\right]$.

The cocycle condition is invariant under Möbius transformations and fixes the functional form of the inhomogeneous term. Furthermore, the cocycle condition fixes the identification

$\mathcal{W}(q)=-\frac{\hbar^{2}}{4 m}\left\{\mathrm{e}^{\left(2 i \mathcal{S}_{0} / \hbar\right)}, q\right\}$

and

$Q(q)=\frac{\hbar^{2}}{4 m}\left\{\mathcal{S}_{0}, q\right\}$

where $\{f, q\}=f^{\prime \prime \prime} / f^{\prime}-\frac{3}{2}\left(f^{\prime \prime} / f^{\prime}\right)^{2}$ denotes the Schwarzian derivative. The cocycle condition, which generalises to higher dimensions [18], implies that $\mathcal{S}_{0}$ is a solution of the quantum stationary HJ equation (QSHJE),

$$
\frac{1}{2 m}\left(\partial_{q} \mathcal{S}_{0}\right)^{2}+V(q)-E+\frac{\hbar^{2}}{4 m}\left\{\mathcal{S}_{0}, q\right\}=0
$$

The equivalence with the one-dimensional stationary version of Eqs. (1) and (2) follows by observing that by Eq. (2) one gets

$$
R=c \frac{1}{\sqrt{\mathcal{S}_{0}^{\prime}}},
$$

with $c$ a non-zero constant, so that

$$
\frac{\Delta R}{R}=-\frac{1}{2}\left\{\mathcal{S}_{0}, q\right\} \text {. }
$$

It is easy to check that the solution of Eq. (6) can be expressed in terms of solutions of the Schrödinger equation. In particular,

$\mathrm{e}^{\frac{2 i}{\hbar} \mathcal{S}_{0}}=\mathrm{e}^{i \alpha} \frac{w+i \bar{\ell}}{w-i \ell}$,

where $\ell=\ell_{1}+i \ell_{2}$, with $\ell_{1}$ and $\ell_{2} \neq 0$ two arbitrary constants, and $w=\psi^{D} / \psi$, with $\psi^{D}$ and $\psi$ two real linearly independent solutions of the Schrödinger equation,

$\left(-\frac{\hbar^{2}}{2 m} \frac{\partial^{2}}{\partial q^{2}}+V(q)-E\right) \psi(q)=0$.

A distinguished feature of the formalism in [11-17] is that both solutions of the Schrödinger equation, $\psi$ and $\psi^{D}$, are kept in the formalism. This can be seen from the properties of the Schwarzian derivative that show that the trivialising transformation is

$q \rightarrow q^{0}=\gamma\left(\psi^{D} / \psi\right)$,

where $\gamma\left(\psi^{D} / \psi\right)$ is an arbitrary Möbius transformation of $\psi^{D} / \psi$. In general, the wave function in the formulation of [11-17] is

$\psi(q)=R(q)\left(A \mathrm{e}^{\frac{i}{\hbar} \mathcal{S}_{0}}+B \mathrm{e}^{-\frac{i}{\hbar} \mathcal{S}_{0}}\right)$.

Furthermore, consistency conditions imply that $\mathcal{S}_{0}(q)$ is never a constant. In particular, the quantum potential $Q(q)$ is never trivial and plays the rôle of intrinsic energy.

The formulation in [11-17] extends to higher dimensions and to the relativistic case as well [18]. Let us now review how energy quantisation arises in our formalism. The QSHJE is equivalent to the equation

$\{w, q\}=-4 m(V(q)-E) / \hbar^{2}$.

This implies that $w \neq$ const, $w \in C^{2}(R)$ and $w^{\prime \prime}$ is differentiable on $\mathbb{R}$. In addition, from the properties of the Schwarzian derivative it follows that 
$\left\{w, q^{-1}\right\}=q^{4}\{w, q\}$

which can be seen as a direct consequence of the cocycle condition. However, such a relation is defined only if the conditions on $w$ hold on the extended real line $\hat{\mathbb{R}}=\mathbb{R} \cup\{\infty\}$. That is, $w \neq$ const, $w \in C^{2}(\hat{\mathbb{R}})$ with $w^{\prime \prime}$ differentiable on $\mathbb{R}$ and

$w(-\infty)= \begin{cases}+w(+\infty) & \text { if } w(-\infty) \neq \pm \infty \\ -w(+\infty) & \text { if } w(-\infty)= \pm \infty\end{cases}$

This means that $w$, and therefore the trivialising map, is a local homeomorphism of $\hat{\mathbb{R}}$ into itself. This implies the continuity of $\left(\psi^{D}, \psi\right)$ and $\left(\psi^{D^{\prime}}, \psi^{\prime}\right)$ without assuming the probability interpretation of the wave function. In particular, the QSHJE is defined only if the ratio $w=\psi^{D} / \psi$ of a pair of real linearly independent solutions of the Schrödinger equation is a local homeomorphism of the extended real line $\hat{\mathbb{R}}=\mathbb{R} \cup\{\infty\}$ into itself. This is an important feature, as the $L^{2}(\mathbb{R})$ condition, which in the Copenhagen formulation is a consequence of the axiomatic interpretation of the wave function, directly follows as a basic theorem which only uses the geometrical gluing conditions of $w$ at $q= \pm \infty$. In particular, denoting by $q_{-}\left(q_{+}\right)$the lowest (highest) $q$ for which $V(q)-E$ changes sign, we have [11-17] the following.

If

$V(q)-E \geq \begin{cases}P_{-}^{2}>0, & q<q_{-}, \\ P_{+}^{2}>0, & q>q_{+},\end{cases}$

then $w=\psi^{D} / \psi$ is a local self-homeomorphism of $\hat{\mathbb{R}}$ iff the Schrödinger equation has an $L^{2}(\mathbb{R})$ solution.

Thus, since the QSHJE is defined if and only if $w$ is a local self-homeomorphism of $\hat{\mathbb{R}}$, this theorem implies that energy quantisation directly follows from the QSHJE itself without further assumptions.

\section{Time parameterisation}

We emphasise that the present approach is fundamentally distinct from the Bohmian one [11-17]. Bohmian mechanics sets

$\psi(q)=R(q) \mathrm{e}^{i S / \hbar}$,

where $\psi$ is the wave function. On the other hand, implementation of the point transformations leading to the trivial hamiltonian necessitates that the wave function is taken in the general form of Eq. (7). Such a condition is reminiscent of the necessity in quantum field theories of using the two solutions of the relativistic quantum equations.

In Bohmian mechanics, time parameterisation is defined by identifying $p$ with the mechanical momentum,

$p=\frac{\partial \mathcal{S}}{\partial q}=m \dot{q}$, with $\mathcal{S}$ a solution of the quantum HJ equation. In classical HJ theory time parameterisation is given by

$t-t_{0}=\frac{\partial \mathcal{S}_{0}^{\mathrm{cl}}}{\partial E}$

which leads to the group velocity. In CM this is equivalent to identifying the conjugate and mechanical momenta. Namely, setting

$p=\frac{\partial \mathcal{S}_{0}^{\mathrm{cl}}}{\partial q}=m \dot{q}$

yields Eq. (11). Bohmian mechanics therefore brings back the notion of trajectories for point particles, since we may solve for $q(t)$. However, we note that the agreement between the definition of time parameterisation of trajectories by Eq. (10) and its definition by the Jacobi theorem (11) is no longer true in quantum mechanics. The use of the latter definition in quantum mechanics, that is,

$t-t_{0}=\frac{\partial \mathcal{S}_{0}}{\partial E}$,

has been first proposed by Floyd [19-27]. In quantum HJ theory this leads to

$m \frac{\mathrm{d} q}{\mathrm{~d} t}=\frac{\partial_{q} \mathcal{S}_{0}}{1-\partial_{E}(V+Q)}$.

Therefore, in quantum mechanics the time parameterisation in Eq. (10) does not coincide with its definition via the Jacobi theorem.

The Floyd proposal (12) would in principle provide a trajectory representation of quantum mechanics which would seem to be in contradiction with the inherently probabilistic nature of quantum mechanics. We show that the definition (12) cannot be implemented. We emphasise, however, that this does not mean that the parameterisation provided by Eq. (12) cannot be useful. In fact, it is quite effective to perform semi-classical approximations. In this respect we note the successful application in studies of molecular dynamics $[9,10]$.

It is clear that in the case of quantised energy spectra Eq. (12) is not defined. On the other hand, the concept of a trajectory should be a universal one, so that one should consider a time parameterisation which is consistent in both cases, discrete and continuous spectra. This essentially would exclude Eq. (12) also in the case of continuous spectra. There is, however, a more stringent argument to show that Eq. (12) cannot be used to define time parameterisation of trajectories. In particular, we now show that the QSHJE is defined only in the case of a discrete spectrum with the case of continuous spectrum arising only as a limiting case. We saw that the cocycle condition led to the QSHJE written as a Schwarzian equation. In particular, we established that the gluing conditions should always hold. Let us now consider the case of a continuous spectrum. Without loss of generality, we may consider 
the case when for large $q$ the potential is zero. For large $q$ two associated real independent solutions of the Schödinger equation are

$\psi=\sin k q, \quad \psi^{D}=\cos k q$.

This means that for large $q$ we have

$w=\tan k q$.

On the other hand, whereas this function at finite distance is a local homeomorphism, it is not the case when considering the entire real axis. This can also be seen by considering a free particle in an infinitely deep well and considering the periodicity conditions. This is equivalent to considering the particle in $S^{1}$ and then sending its radius $R$ to infinity. For any finite $R$ the ratio of two linearly independent solutions is a local homeomorphism of $S^{1}$ and the spectrum is discrete. In the $R \rightarrow \infty$ limit the gluing condition is no longer under control. In the case of the free particle in an infinitely deep potential well of width $L$, the energy levels are

$E_{n}=\frac{\hbar^{2} \pi^{2}}{2 m L^{2}} n^{2}$,

$n \in \mathbb{N}_{+}$. It follows that for any finite value of $L$ the spectrum is discrete. We then conclude that imposing the cocycle condition requires a discrete spectrum, which in turn implies that continuous variation of the energy is not possible. Equivalently, the time parameterisation by the Jacobi theorem is not well defined. This can be generalised to the free particle in any space dimension, leading to the same conclusion. On the other hand, it is a general theorem that the equation

$-\frac{\hbar^{2}}{2 m} \Delta \psi=E \psi$,

in bounded domains of $\mathbb{R}^{n}$, therefore including e.g. the $(n-1)$-sphere $S^{n-1}$, has only a discrete spectrum. At the level of the quantum $\mathrm{HJ}$ equation, this is just a consequence of the non-triviality of the quantum potential.

Therefore, without using any axiomatic interpretation of the wave function, we have shown that trajectories cannot be derived from the quantum $\mathrm{HJ}$ equation. In particular, the concept of a localised particle with a well-defined velocity does not exist. Since trajectories do not exist in the formulation of [11-17], one may try to consider finite differences instead of derivatives,

$t_{n}=\frac{\mathcal{S}_{0}\left(E_{n+1}\right)-\mathcal{S}_{0}\left(E_{n}\right)}{E_{n+1}-E_{n}}$.

This is a basic point since it leads one to consider the superposition of different energy eigenstates, which is at the basis of the interference phenomena. In particular, whereas time evolution of a hamiltonian eigenfunction corresponds to an overall phase, so that it describes the same ray vector in the
Hilbert space, the role of time is apparent, through interference, just when the physical system is the superposition of at least two energy levels. Equation (13) indicates that the interference phenomena are deeply related to the concept of time.

Whereas Eq. (13) sheds light on the origin of interference in the quantum Hamilton-Jacobi formulation, there is an interesting alternative which is suggested by the Schrödinger equation. One may think that time parameterisation may be defined as a sort of random parameter whose distribution is fixed by the properties of the distribution of the energy eigenvalues. In other words, instead of considering a finite difference, which gives $t=t(E)$, we may search for a more intrinsic definition. The idea is that the wave function depends on $E$, so one may formally invert this relation to $E=E(\psi)$. This leads us to consider $t_{\psi}=t(\psi)$. In particular, consider a time-independent potential. In this case the Schrödinger equation implies

$\psi(q, t)=\sum_{k} c_{k} \mathrm{e}^{-\frac{i}{\hbar} E_{k} t} \psi_{k}(q)$,

where the $\psi_{k}$ are the hamiltonian eigenfunctions

$H \psi_{k}=E_{n} \psi_{k}$.

Next, recall that for one-dimensional bound states $\psi_{n}$ can be normalised in such a way that it takes real values. Multiplying Eq. (14) by $\psi_{n}$ and integrating over $\mathbb{R}$ one gets

$t_{\psi}=\frac{i \hbar}{E_{n}}\left(\ln \int_{\mathbb{R}} \psi \psi_{n} \mathrm{~d} q-\ln \int_{\mathbb{R}} \psi(q, 0) \psi_{n} \mathrm{~d} q\right)$.

Note, however, that $t_{\psi}$ is just the time experienced by the observer. This suggests that there exists a kind of time parameterisation leading to the probabilistic interpretation of trajectories in the framework of the quantum $\mathrm{HJ}$ equation.

\section{Conclusions}

Understanding the synthesis of quantum mechanics and general relativity remains the pivotal goal of fundamental physics. The main effort in this endeavour is in the framework of string theory. String theory provides a self-consistent perturbative approach to quantum gravity. The main achievement of string theory is that it gives rise to the gauge and matter ingredients of elementary particle physics, and it predicts the number of degrees of freedom required to obtain a consistent theory. String theory therefore enables the development of a phenomenological approach to quantum gravity. The state of the art in this regard is the construction of Minimal Standard Heterotic String Models [31-35]. Over the past few years important progress has also been achieved in the understanding of the perturbative expansion of string 
theory for genus $g$ Riemann surfaces; see for example [36$45]$ and references therein. Despite its success, string theory does not provide a conceptual starting point for formulating quantum gravity. What we seek is a fundamental hypothesis of which, possibly, string theories arise as perturbative limits. A basic property of string theory is T-duality [46], which may be viewed as phase-space duality in compact space.

In this paper we examined the definability of time parameterisation of trajectories in the framework of the quantum $\mathrm{HJ}$ equation. We first observed that it is not possible to perform infinitesimal variations of the energy when this is quantised. As such, the quantum HJ equation does not lead to the concept of trajectory. Next, we discussed the identification of the mechanical momentum with the conjugate momentum,

$m \dot{q}=\frac{\partial \mathcal{S}}{\partial q}$

as done in Bohmian mechanics, and we noticed that it is inconsistent with its derivation from Jacobi's theorem, which in turn cannot be applied in the case of systems with quantised energy. On the other hand, consistency arguments show that if trajectories cannot be defined in the case of quantised energy, then trajectories do not exist even when the energy spectrum is continuous.

In this respect it should, however, be stressed that quantum trajectories provide a powerful approximation tool in quantum dynamics $[9,10,47]$. In particular, the method of quantum trajectories has been developed as a computational tool to solve time-dependent quantum mechanical problems by evolving ensembles of correlated quantum trajectories through the integration of the hydrodynamic equations. These quantum trajectories serve as a computational adaptive moving grid, and from this perspective these are not authentic trajectories.

We then proposed that the above features of the quantum $\mathrm{HJ}$ equation are at the heart of the probabilistic nature of quantum mechanics. In this respect, we considered time as dependent on the wave function, getting an expression that suggests considering time itself as having a probabilistic nature.

We also observed that the derivation of the quantum $\mathrm{HJ}$ equation from the cocycle condition [11-17] is naturally formulated in compact spaces that can be extended considering the decompactification limit. This suggests that our space is compact. In this case all the possible energy spectra are quantised and would make the Dirac and von Neumann formulations of quantum mechanics essentially equivalent. The compactness of space has basic observational consequences and forthcoming evidence for it may exist in the cosmic microwave background radiation [48].
Acknowledgments AEF is supported by STFC under contract ST/J0 00493/1. MM is supported by the Padova University Project CPDA1193 49 and by the MIUR-PRIN contract 2009-KHZKRX.

Open Access This article is distributed under the terms of the Creative Commons Attribution License which permits any use, distribution, and reproduction in any medium, provided the original author(s) and the source are credited.

Article funded by $\mathrm{SCOAP}^{3}$ and licensed under CC BY 4.0

\section{References}

1. V. Periwal, Phys. Rev. Lett. 80, 4366 (1998)

2. M.H. Partovi, Phys. Rev. Lett. 89, 144101 (2002)

3. D. Dürr, S. Goldstein, R. Tumulka, N. Zanghì, Phys. Rev. Lett. 93, 090402 (2004)

4. C. Meier, Phys. Rev. Lett. 93, 173003 (2004)

5. M. Roncadelli, L.S. Schulman, Phys. Rev. Lett. 99, 170406 (2007)

6. C.-C. Chou, A.S. Sanz, S. Miret-Artés, R.E. Wyatt, Phys. Rev. Lett. 102, 250401 (2009)

7. W. Koch, F. Grossmann, D.J. Tannor, Phys. Rev. Lett. 105, 230405 (2010)

8. B. Vakili, N. Khosravi, Phys. Rev. D 85, 083529 (2012)

9. R.E. Wyatt, Quantum dynamics with trajectories: introduction to quantum hydrodynamics (Springer, Berlin, 2005)

10. B. Poirier, J. Chem. Phys. 121, 4501 (2004)

11. A.E. Faraggi, M. Matone, Phys. Lett. B 450, 34 (1999)

12. A.E. Faraggi, M. Matone, Phys. Lett. B 437, 369 (1998)

13. A.E. Faraggi, M. Matone, Phys. Lett. A 249, 180 (1998)

14. A.E. Faraggi, M. Matone, Phys. Lett. B 445, 77 (1998)

15. A.E. Faraggi, M. Matone, Phys. Lett. B 445, 357 (1998)

16. A.E. Faraggi, M. Matone, Int. J. Mod. Phys. A 15, 1869 (2000)

17. A.E. Faraggi, M. Matone, Contribute to the book Quantum Trajectories, ed. P. Chattaraj, Taylor \& Francis, Oxford (2011). (arXiv: 0912.1225)

18. G. Bertoldi, A.E. Faraggi, M. Matone, Class. Quantum Gravity 17, 3965 (2000)

19. E.R. Floyd, Phys. Rev. D 25, 1547 (1982)

20. E.R. Floyd, Phys. Rev. D 26, 1339 (1982)

21. E.R. Floyd, Phys. Rev. D 29, 1842 (1984)

22. E.R. Floyd, Phys. Rev. D 34, 3246 (1986)

23. E.R. Floyd, Found. Phys. Lett. 9, 489 (1996)

24. E.R. Floyd, Found. Phys. Lett. 13, 235 (2000)

25. E.R. Floyd, Phys. Lett. A 214, 259 (1996)

26. E.R. Floyd, Int. J. Mod. Phys. A 14, 1111 (1999)

27. E.R. Floyd, Int. J. Mod. Phys 15, 1363 (2000)

28. A.E. Faraggi, M. Matone, Phys. Rev. Lett. 78, 163 (1997)

29. J. von Neumann, Mathematical foundations of quantum mechanics. Princeton University Press, Princeton (1996)

30. P.A.M. Dirac, The Principles of Quantum Mechanics. Oxford University Press, Oxford (1988)

31. A.E. Faraggi, D.V. Nanopoulos, K. Yuan, Nucl. Phys. B 335, 347 (1990)

32. A.E. Faraggi, Phys. Lett. B 278, 131 (1992)

33. A.E. Faraggi, Nucl. Phys. B 387, 239 (1992)

34. G.B. Cleaver, A.E. Faraggi, D.V. Nanopoulos, Phys. Lett. B 455, 135 (1999)

35. K. Christodoulides, A.E. Faraggi, J. Rizos, Phys. Lett. B 702, 81 (2011)

36. M. Matone, R. Volpato, Nucl. Phys. B 732, 321 (2006)

37. M. Matone, R. Volpato, Nucl. Phys. B 806, 735 (2009)

38. M. Matone, R. Volpato, Nucl. Phys. B 839, 21 (2010)

39. M. Matone, R. Volpato, Proc. Am. Math. Soc. 141, 2575 (2013)

40. M. Matone, R. Volpato, Math. Ann. 355, 327 (2013) 
41. M. Matone, R. Volpato, J. Math. Phys. 52, 102305 (2011)

42. M. Matone, Int. J. Mod. Phys. A 10, 289 (1995)

43. M. Matone, JHEP 1210, 175 (2012)

44. M. Matone, JHEP 1211, 050 (2012)
45. M. Matone, Phys. Rev. D 89, 026008 (2014)

46. A. Giveon, M. Porrati, E. Rabinovici, Phys. Rep. 244, 77 (1994)

47. R.E. Wyatt, J. Chem. Phys. 117, 9569 (2002)

48. G. Aslanyan, A.V. Manohar, JCAP 1206, 003 (2012) 